\title{
An Analysis of Post-Pacing R-R Intervals During Atrial Fibrillation
}

\author{
FRED MORADY, LORENZO A. DICARLO, JR., RYSZARD B. KROL, \\ MICHAEL DE BUITLEIR, and JEFFERY M. BAERMAN \\ From the Division of Cardiology, University of Michigan Medical Center, Ann Arbor, Michigan
}

MORADY, F., ET AL.: An analysis of post-pacing R-R intervals during atrial fibrillation. Bursts of ventricular pacing at cycle lengths of 350-260 ms were introduced during atrial fibrillation in nine patients, and the post-pacing R-R intervals were compared to the R-R intervals of spontaneous QRS complexes. In eight of nine patients, the mean post-pacing R-R interval was 126-199 ms longer than the mean spontaneous $R-R$ interval $(p<0.005)$.

Spontaneous runs of aberrantly conducted supraventricular complexes were recorded during atrial fibrillation in one patient. The mean R-R interval following the runs of aberrantly conducted supraventricular complexes was significantly longer than the mean R-R interval of spontaneous narrow QRS complexes ( $p<0.001)$, but not significantly different than the mean post-pacing $R-R$ interval.

The findings of this study suggest that the R-R interval that follows a wide-complex tachycardia during atrial fibrillation is unlikely to be of value in differentiating ventricular tachycardia from aberrantly conducted supraventricular complexes. Analysis of R-R intervals that follow bursts of ventricular pacing suggests that there is likely to be considerable overlap between the $R-R$ intervals that follow runs of ventricular tachycardia and the spontaneous $\mathrm{R}-\mathrm{R}$ intervals during atrial fibrillation. Furthermore, even when the posttachycardia R-R interval clearly exceeds the longest spontaneous R-R interval during atrial fibrillation, this is still of little diagnostic value, because a long pause may occur after either a run of ventricular tachycardia or a run of aberrantly conducted QRS complexes of supraventricular origin. (PACE, Vol. 9, May-June, 1986)

atrial fibrillation, ventricular tachycardia, aberration

\section{Introduction}

It has been demonstrated that premature ventricular complexes during atrial fibrillation are followed by R-R intervals that are longer than the $\mathrm{R}-\mathrm{R}$ intervals between narrow QRS complexes. ${ }^{1-3}$ Lengthening of the R-R interval after a premature ventricular complex has been attributed to concealed retrograde penetration of the atrioventricular junction. ${ }^{2}$ The presence of a long pause after a wide QRS complex during atrial fibrillation has been described as being of diagnostic value in distinguishing a premature ventricular complex from an aberrantly conducted supraventricular complex. $^{1,4}$

Address for reprints: Fred Morady, M.D., Division of Cardiology, Rm. W-11511, University Hospitals, 1405 East Ann Street, Ann Arbor, MI 48109.

Received August 5, 1985; revision received October 29, 1985; accepted October 29, 1985.
In regard to wide-complex tachycardia in the setting of atrial fibrillation, the diagnostic value of the post-tachycardia R-R interval in differentiating ventricular tachycardia from aberrantly conducted supraventricular complexes has; to date, not been investigated in a systematic fashion. Because the last ventricular depolarization in a run of ventricular tachycardia may penetrate the atrioventricular junction to a variable degree, the $\mathrm{R}-\mathrm{R}$ interval following an episode of ventricular tachycardia during atrial fibrillation may not always be longer than the spontaneous R-R intervals.

In this study, ventricular tachycardia was simulated by bursts of ventricular pacing during atrial fibrillation. The purpose of this study was to compare the post-pacing R-R intervals to the R$\mathrm{R}$ intervals of spontaneous QRS complexes, to determine whether the post-tachycardia R-R interval may be of diagnostic value in differentiating ventricular tachycardia from aberrantly conducted supraventricular complexes during atrial fibril- 
Table I.

Spontaneous and Post-Pacing R-R Intervals During Atrial Fibrillation

\begin{tabular}{|c|c|c|c|c|c|}
\hline \multirow[b]{2}{*}{ Patient } & \multicolumn{2}{|c|}{$\begin{array}{l}\text { R-R Intervals of Spontaneous } \\
\text { Complexes }\end{array}$} & \multicolumn{2}{|c|}{$\begin{array}{l}\text { R-R Intervals Following } \\
\text { Ventricular Pacing }\end{array}$} & \multirow[b]{2}{*}{ p Value } \\
\hline & mean $\pm S D(m s)^{*}$ & range (ms) & mean $\pm \mathrm{SD}(\mathrm{ms})^{*}$ & range (ms) & \\
\hline 1 & $525 \pm 126$ & $360-840$ & $714 \pm 150$ & $360-1120$ & $<0.001$ \\
\hline 2 & $482 \pm 48$ & $380-580$ & $681 \pm 139$ & $400-880$ & $<0.001$ \\
\hline 3 & $614 \pm 127$ & $440-940$ & $853 \pm 83$ & $680-1080$ & $<0.001$ \\
\hline 4 & $839 \pm 181$ & $520-1280$ & $1027 \pm 200$ & $400-1520$ & $<0.001$ \\
\hline 5 & $653 \pm 158$ & $400-1120$ & $643 \pm 193$ & $280-1200$ & $>0.05$ \\
\hline 6 & $870 \pm 198$ & $520-1280$ & $1051 \pm 232$ & $560-1640$ & $<0.001$ \\
\hline 7 & $419 \pm 95$ & $280-660$ & $545 \pm 83$ & $400-720$ & $<0.001$ \\
\hline 8 & $492 \pm 95$ & $360-760$ & $645 \pm 172$ & $320-920$ & $<0.001$ \\
\hline 9 & $480 \pm 110$ & $320-760$ & $654 \pm 123$ & $320-880$ & $<0.005$ \\
\hline
\end{tabular}

* Sample size in each patient is 50 R-R intervals.

$\mathrm{SD}=$ standard deviation

lation. In one patient who had spontaneous runs of aberrantly conducted supraventricular complexes during atrial fibrillation, it was possible to compare the R-R intervals following the aberrantly conducted complexes with the R-R intervals following bursts of ventricular pacing.

\section{Methods}

The subjects in this study were nine patients with chronic or recurrent atrial fibrillation who underwent electrophysiologic testing for evaluation of sustained ventricular tachycardia or unexplained syncope. There were seven men and two women, and their mean age was $59 \pm 14$ years (mean \pm one standard deviation). Five patients had coronary artery disease, two had a dilated cardiomyopathy, and two had no identifiable structural heart disease. No patient had a bundle branch block.

After informed consent was obtained, an electrophysiologic study was performed in the fasting, unsedated state, at least four half-lives after discontinuation of all antiarrhythmic drugs, including digitalis. One to three electrode catheters were inserted through a femoral vein and positioned in the right atrium, across the tricuspid valve, or in the right ventricle, as clinically indicated. After completion of the clinical portion of the electrophysiologic study in the drug-free state, the study protocol was performed. Chronic atrial fibrillation was present in six patients; in the remaining three patients, sustained atrial fibrillation was induced by rapid atrial pacing.

A quadripolar electrode catheter was positioned against the apex of the right ventricle. Electrocardiographic leads $\mathrm{V}_{1}, \mathrm{I}$, and III, and an intracardiac electrogram from the right ventricular apex were recorded on an Electronics-for-Medicine* VR-12 recorder at a paper speed of $25-50$ $\mathrm{mm} / \mathrm{s}$.

After a five-minute rest period, a one-minute recording of atrial fibrillation was obtained. Ventricular pacing was then performed at a current strength of twice diastolic threshold with stimuli $2 \mathrm{~ms}$ in duration, using a programmable stimulator (Bloom Associates, Ltd.**). Trains of nine stimuli were introduced at the right ventricular apex at cycle lengths of $350-260 \mathrm{~ms}$, in 10-ms decrements. Pacing at each cycle length was performed five times, for a total of 50 trains. The inter-train interval was 8 seconds. Upon completion of the pacing protocol, an additional one-minute recording of atrial fibrillation was obtained.

In each patient, the interval between the last paced ventricular complex of the 50 pacing trains and the next spontaneous QRS complex was measured. Also measured were $50 \mathrm{R}-\mathrm{R}$ intervals

\footnotetext{
* Electronics-for-Medicine, Pleasantville, NY, USA.

** Bloom Associates, Ltd., Narbeth, PA, USA.
} 
between spontaneous narrow QRS complexes (25 consecutive R-R intervals recorded before ventricular pacing, and 25 afterwards). In one patient (no. 2), 21 spontaneous runs of aberrantly conducted supraventricular complexes during atrial fibrillation were recorded, and the intervals between the last aberrantly conducted complex and the next narrow QRS complex were measured.

Post-pacing R-R intervals were compared to spontaneous R-R intervals during atrial fibrillation using Student's $t$ test. In patient no. 2, the R-R intervals between narrow QRS complexes, postpacing R-R intervals, and R-R intervals following runs of aberrantly conducted supraventricular complexes were compared by analysis of variance.

\section{Results}

The mean R-R interval of spontaneous narrow QRS complexes and the mean post-pacing R-R interval during atrial fibrillation in each of the nine patients are tabulated in Table I. In eight patients, the mean post-pacing R-R interval was 126-199 $m$ s longer than the mean R-R interval of spontaneous QRS complexes ( $<<0.001-0.005$, Table I). In one patient (no. 5), there was not a significant difference between the mean R-R interval of spontaneous QRS complexes and the mean postpacing R-R interval ( $p>0.05$ ). There was not an association between the post-pacing $\mathrm{R}-\mathrm{R}$ interval and the pacing cycle length.

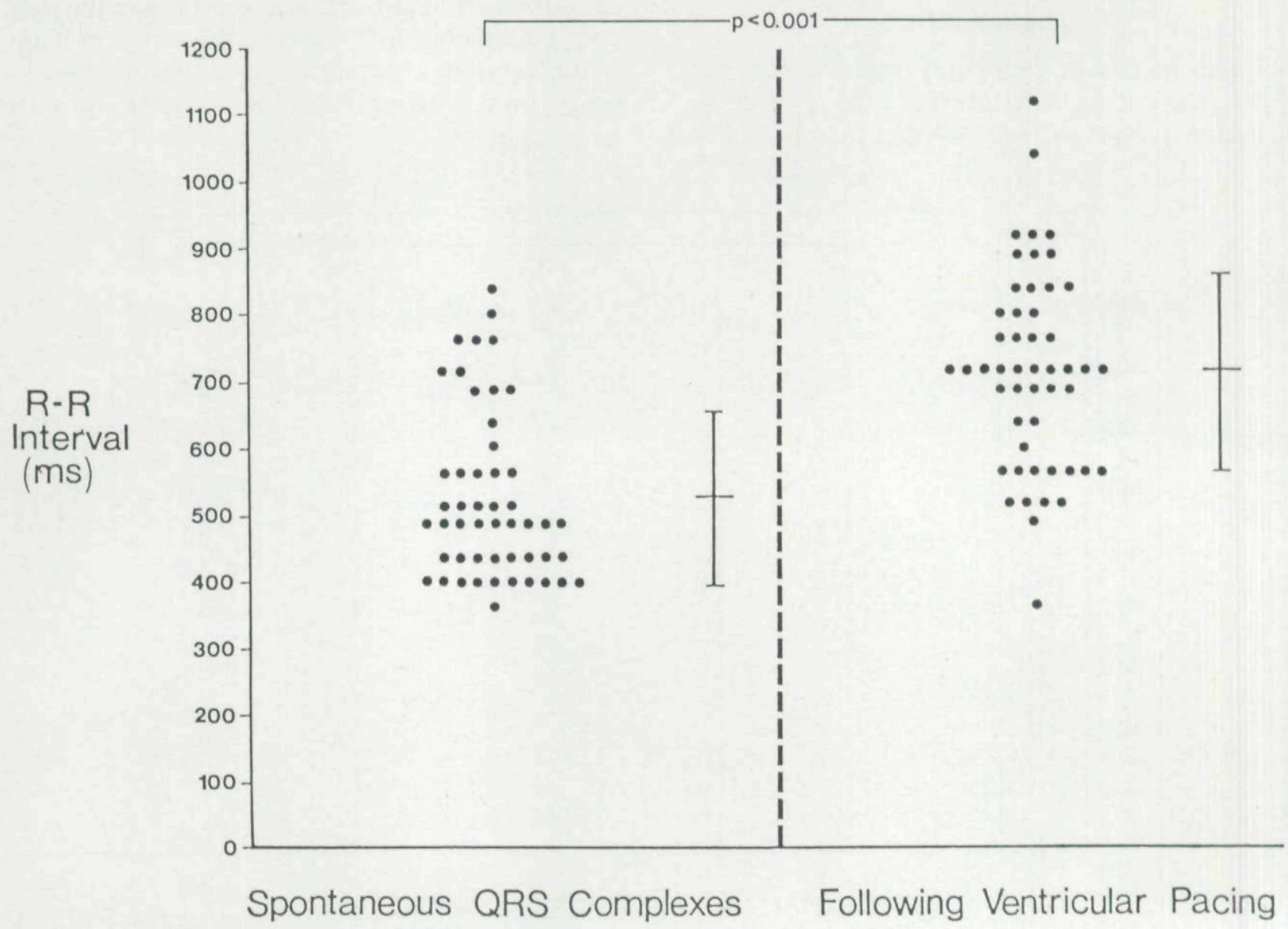

Figure 1. The spontaneous $R-R$ intervals during atrial fibrillation $(n=50)$ and the post-pacing $R-R$ intervals $(n=50)$ in patient no. 1 . Although the mean post-pacing $R-R$ interval is significantly longer than the mean spontaneous R-R interval, there is considerable overlap between the two groups of R-R intervals. Only $16 \%$ of post-pacing R-R intervals exceeded the longest spontaneous $\mathrm{R}-\mathrm{R}$ interval. 
In eight patients, there was considerable overlap between the spontaneous R-R intervals and the post-pacing R-R intervals (Fig. 1). Excluding patient no. 2, the percentage of post-pacing $\mathrm{R}$ $\mathrm{R}$ intervals that exceeded the longest recorded spontaneous R-R interval varied between $4-22 \%$.

Patient no. 2 had spontaneous runs of aberrantly conducted supraventricular complexes. The mean R-R interval following a run of aberrantly conducted supraventricular complexes, $711 \pm 132$ $m s$, was significantly longer than the mean R-R interval between spontaneous narrow QRS complexes, $482 \pm 48 \mathrm{~ms}(\mathrm{p}<0.001)$, but was not significantly different than the mean post-pacing $\mathrm{R}$ $R$ interval, $681 \pm 139 \mathrm{~ms}$ ( $p>0.05$ ) (Figs. 2 and 3 ).

\section{Discussion}

The results of this study indicate that the mean post-pacing $\mathrm{R}-\mathrm{R}$ interval during atrial fibrillation in most patients is longer than the mean
R-R interval of spontaneous QRS complexes. Therefore, if a wide-complex tachycardia is followed by an R-R interval longer than any of the spontaneous R-R intervals, this may suggest that the tachycardia is ventricular in origin. However, analysis of post-pacing intervals suggests that only a small proportion of episodes of ventricular tachycardia are likely to be followed by an R-R interval which exceeds the longest spontaneous R-R interval. Moreover, observations made in patient no. 2 indicate that runs of aberrantly conducted supraventricular complexes may also be followed by an R-R interval which exceeds the longest spontaneous $\mathrm{R}-\mathrm{R}$ interval recorded during atrial fibrillation. Therefore, even when the R-R interval which follows a wide-complex tachycardia during atrial fibrillation is longer than the longest spontaneous R-R interval, this is not of diagnostic value in differentiating ventricular tachycardia from aberrantly conducted supraventricular complexes.

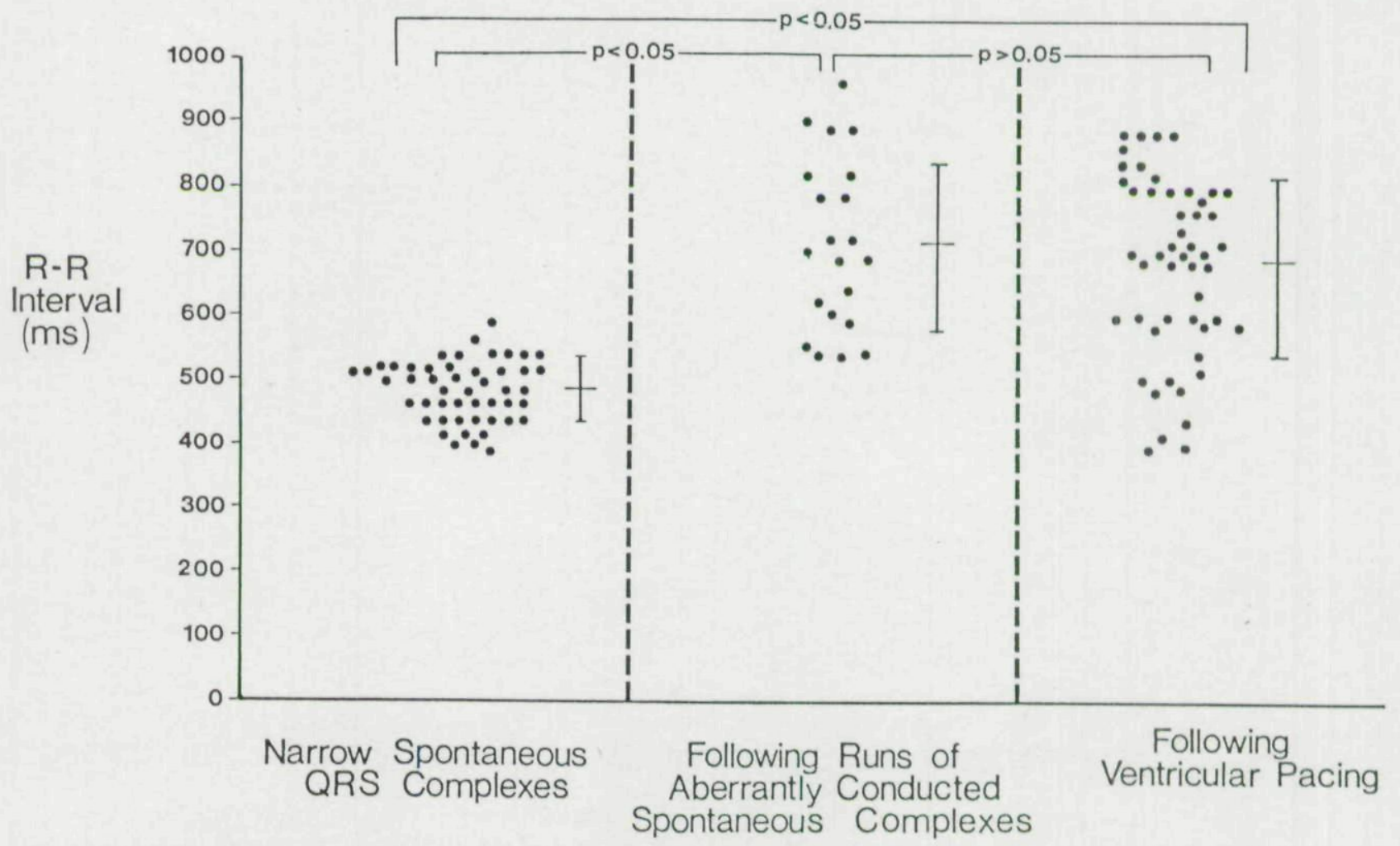

Figure 2. The R-R intervals of spontaneous narrow QRS complexes ( $n=50$ ), the $R-R$ intervals following runs of aberrantly conducted QRS complexes of supraventricular origin $(n=21)$, and the post-pacing $R-R$ intervals $(n=50)$ during atrial fibrillation in patient no. 2 . 
A.

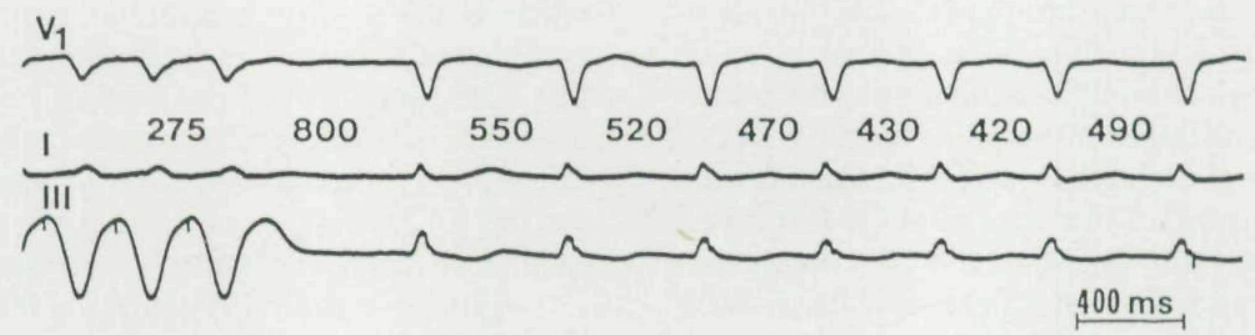

B.

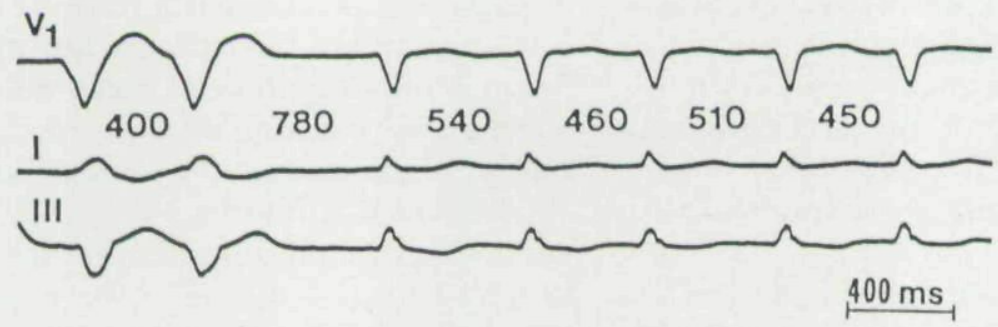

C.
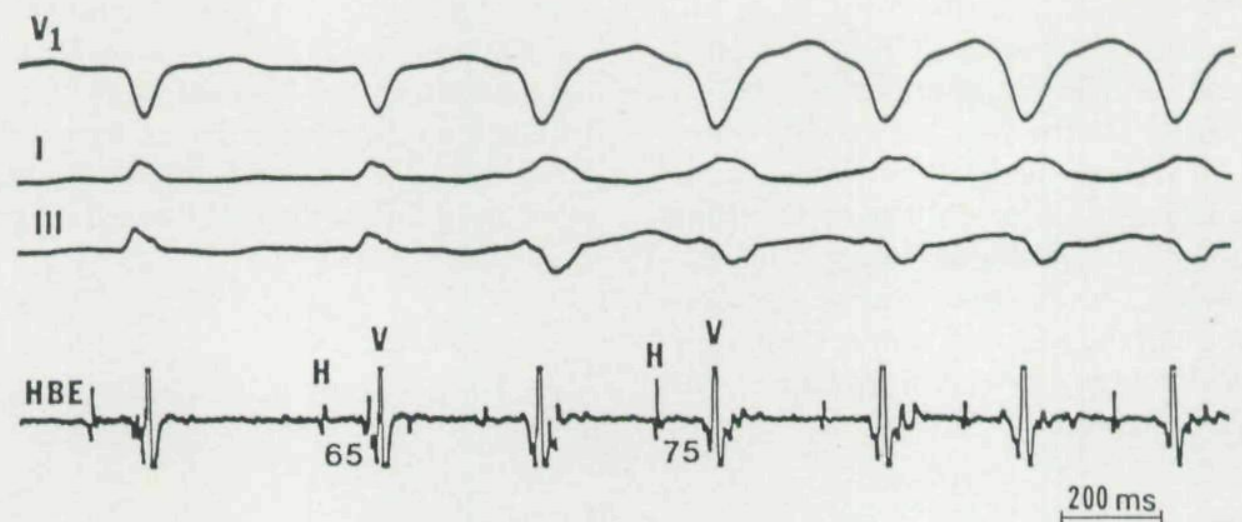

Figure 3. Recordings of electrocardiographic leads $V_{1}, I$, and III during atrial fibrillation, in patient no. 2. The numbers above lead I refer to the R-R intervals, in ms. A: On the left are the last three QRS complexes resulting from a train of nine paced stimuli at a cycle length of $275 \mathrm{~ms}$. The post-pacing R-R interval is $800 \mathrm{~ms}$, which exceeds the R-R intervals of spontaneous narrow QRS complexes (range 380-580 ms). B: On the left are the last two QRS complexes of a run of five aberrantly conducted QRS complexes of supraventricular origin, cycle length $360-400 \mathrm{~ms}$. The aberrantly conducted complexes, which have a left bundle branch block configuration, are followed by an R-R interval of $780 \mathrm{~ms}$, which also exceeds the range of R-R intervals of spontaneous narrow QRS complexes. C: A His bundle electrogram (HBE) demonstrates that the wide QRS complexes which have a left bundle branch block configuration in Figure 1 are preceded by a bundle of His depolarization $(\mathrm{H})$ and therefore are aberrantly conducted QRS complexes of supraventricular origin. The infranodal conduction time of the narrow and wide QRS complexes is $65 \mathrm{~ms}$ and 75 ms, respectively. 
Pritchett et al. demonstrated that premature ventricular complexes induced during atrial fibrillation were followed by a cycle length which was 107-136 ms longer than the mean control cycle length. ${ }^{3}$ Similarly, in the present study, bursts of ventricular pacing during atrial fibrillation were followed in most patients by a mean R-R interval which was 126-199 ms longer than the mean control R-R intervals. The lengthening of the R-R interval which may occur after either a single premature ventricular complex or a train of paced ventricular complexes is presumably caused by retrograde penetration of the atrioventricular junction. In our patient no. 5 , in whom there was not a lengthening of the post-pacing R-R interval, it may be that there was no retrograde penetration of the atrioventricular junction. Because chronic atrial fibrillation was present in this patient, it was not possible to assess ventriculo-atrial conduction during sinus rhythm.

An unexpected finding of this study was a lengthening of the R-R interval following runs of aberrantly conducted supraventricular complexes in patient no. 2. The degree of lengthening of the $R-R$ interval was equal in magnitude to the lengthening of the post-pacing R-R intervals. A possible explanation for this phenomenon is that with the aberrantly conducted complexes, which had a left bundle branch block configuration, there was anterograde conduction only over the right bundle; this may have allowed retrograde conduction up the left bundle and concealed retrograde penetration of the atrioventricular junction, simulating the effects of ventricular pacing on the atrioventricular junction.

\section{References}

1. Langendorf, R.: Aberrant ventricular conduction. Am. Heart J., 41:700, 1951.

2. Langendorf, R., Pick, A.: Artifical pacing of the human heart. Its contribution to the understanding of arrhythmias. Am. J. Cardiol., 28:516, 1971.
A limitation of this study is that spontaneous runs of aberrantly conducted supraventricular complexes occurred in only one patient. Therefore, the incidence of the type of response noted in patient no. 2, in whom there was a lengthening of the R-R interval after runs of aberrantly conducted QRS complexes, cannot be estimated. A second possible limitation is that pacing was performed only in the right ventricle. It is not known whether left ventricular pacing may have had a different effect on the atrioventricular junction and, therefore, a different effect on the post-pacing $\mathrm{R}-\mathrm{R}$ interval.

In conclusion, the R-R interval which follows a wide-complex tachycardia during atrial fibrillation is unlikely to be of diagnostic value in differentiating ventricular tachycardia from aberrantly conducted supraventricular complexes. Analysis of R-R intervals which follow bursts of ventricular pacing suggests that there is likely to be a large degree of overlap between the R-R intervals that follow runs of ventricular tachycardia and the spontaneous R-R intervals during atrial fibrillation. Furthermore, even if the post-tachycardia R-R interval is clearly longer than the longest spontaneous R-R interval, this is still of little diagnostic value, because runs of aberrantly conducted supraventricular complexes may be followed by R-R intervals which are as long as those that may follow runs of ventricular tachycardia.

Acknowledgment: The authors gratefully acknowledge the excellent secretarial assistance of Lisa Hackbarth.

3. Pritchett, E.L.C., Smith, W.M., Klein, G.J., et al.: The "compensatory pause" of atrial fibrillation. Circulation, 62:1021, 1980.

4. Chung, E.K.: Principles of Cardiac Arrhythmias. Baltimore, Williams \& Wilkins, 1977, p. 97-188. 
This document is a scanned copy of a printed document. No warranty is given about the accuracy of the copy. Users should refer to the original published version of the material. 